\title{
Cloud-Based Access Control Framework for Effective Role Provisioning in Business Application
}

\author{
Auxilia M., Research Scholar, Sathayabama University, Chennai, India \\ Raja K., Dhaanish Ahmed College of Engineering, Chennai, India \\ Kannan K., AudiSankara College of Engineering and Technology, Gudur, Nellore, India
}

\begin{abstract}
In the evolution of social networks and big data, secure information sharing is a crucial task. When information is shared between the user and the organization admin, security plays a key role in any business organization in terms of privacy. Though many fruitful solutions prevail to protect the data integrity and privacy, there is a huge space for novel data protection schemes where a large set of data are involved. In this article, the Cloud-Based Access Control (C-BAC) framework is proposed which can fit in any business organization application. In this C-BAC, Policy Enforcement Point (PEP) is used to avoid unwanted information sharing with the neighboring employee. C-BAC framework with RSA provides security, based on the number of employees with the data handled by the particular employee, better than the existing access control framework with asymmetric encryption standard (AES) and Rivest-Shamir-Adleman (RSA) in terms of individual information handling.
\end{abstract}

\section{KEYWORDS}

Aggregate Zero-Knowledge Proof Knowledge, Information Sharing, Oblivious Commitment-Based Envelope, Policy Enforcement Point, Role Based Access Control, Security

\section{INTRODUCTION}

In recent days, web services are performing various computations on several scenarios like networks, big data and cloud computing, etc., where ambient computing plays a vital role. It acts as a framework to control several sets of services, resources to utilize it efficiently and seamlessly to any scenario. It also helps in making the services user-friendly and effective and it supports improved user interaction when the particular service is utilized for the users. Nowadays, resources are offered and utilized in large quantity, thus ambient computing plays an important role in cloud-based services.

Cloud Services in the web has been growing and attracting the attention of researchers based on issues like uncertainty during the authentication of Cloud services, lack of decision-making process during the authorization of services and information loss during confidential information exchange. World Wide Web plays a vital role in this current trend related to the concept of cloud technology. For every business organization, role provisioning is the initial step for every employee (Chandrashekar et al., 2017; Jonathan \& Dusit, 2017). Every role provisioning system must have certain conditions to assign an appropriate role to the employee (Ilia \& Rizos, 2016; Jonathan \& Dusit, 2017). In general condition, ' $\mathrm{R}$ ' provides a condition for each role assigned to all employees in the organization. The role of enrolment is done based on the concept of Role Based Access Control (RBAC) (Yiqun et al., 
2008) which is used to assign a role in a suitable way. Then each employee must be assigned and allowed to use several services based on a particular application by integrating with the workflow foundation (Ilia, 2016). Workflow is a sequence of the inter-connected process which contains a set of operations that include work assigned to a particular person or a group of persons or a set of persons in an organization or a mechanism having a simple or complex procedure to perform. (Ward et al., 2015). As all know, large sets of information are in existence in all other domains including networks, data mining (Santosh, 2018) Big data (Feldiansyah, 2018) and machine learning (Lazaros \& Ahmad, 2017; Brian, 2018), etc.

For the execution of the above workflow, scheduling the task is needed and several methodologies of the workflow are detailed as 1 . The work process is executed based on calling a thread which is not generated especially for workflow process which means that the process calling has to wait until the workflow completes its process by the invoker. 2 . When the work process executes its method using a new thread by the application. At the same time, the executed process calling will be paused. 3. Window Communication Foundation (WCF) will be served when the work process executes its method. The resultant work process which is served will use the data as input from the network which contains the activities of the host service. There are two condition policies, which are applied during the workflow execution. There should be a fixed condition for a certain role in an organization. Aggregate Zero Knowledge Proof knowledge (AgZKPk) protocol (Uwe et al., 2008) is used to provide privacy and authentication. There will be no fixed condition and the conditions can be framed as an employee identity attributes. Oblivious Commitment Based Envelope (OCBE) (Jiangtao et al., 2006) protocol is used for these situations.

The situations in OCBE are applied based on certain conditions, condition 1 to condition $\mathrm{N}$ which are identified as attributes. These attributes are framed based on employee information. Their information consists of identity attributes and conditions for several employees (Yiqun et al., 2008). For example, "social security number", "birth-date" and "employment" are represented as attributes which are unique. In this paper, services are concerned with the deployment of the proposed cloudbased business framework with better improvement, in terms of cost, execution time and slack deadline based on the number of resources utilized and the performance of the proposed C-BAC is analyzed based on different security levels.

The organization of this paper is as follows: several current research work solutions are explained in the 'Related Works' section. In the next section 'C-BAC Role Provisioning Framework', C-BAC framework is deployed based on role provisioning and its working prototype is briefed. Our proposed model is analyzed under varied parameters and is discussed in the 'Implementation and Performance Analysis' section. Finally, our conclusions along with future directions are presented in the last section.

\section{RELATED WORKS}

Federica Paci et al. (2008) proposed Business Process Execution Language (BPEL) with RBAC framework based on employee information as an attribute and provide privacy based on two protocols and cryptographic algorithm (AES) (Federica et al., 2008). Mainly, PEP acts as privacy tools to provide security in an organization (Kalliopiet et al., 2016). Shenhai-bo and Hong Fan discuss the attribute-based access control model which has granted access to serve based on an attribute of inter-related entities which will be considered as the sensitive attributes (Hai-bo et al., 2006). They provide access control based on identity-based authorization and to protect user's privacy. Cloud services which contain insecurely application will be identified and executed through the internet without authentication. It is quite different with respect to protecting our system like files or document which contains executable software components which will be served as per the request set as an input parameter. To develop an effective access control model based on dynamic cloud-based XML in web service, the access control model will capture the information related to time, location based on the time where the request and control decision are accessed. Sheng et al. (2009) proposed tools 
to provide adaptive service composition and provisioning in the Child Care Assistance Program (CCAP) and they have also provided an RBAC model. There are certain configuring service contexts and exceptions are provided to accommodate different users.

As the BPEL model helps to provide services through the web, there is a possibility of integrating other domain processes to extend the services in other aspects (Hooman \& Seyed, 2019). Here the access control model is integrated with the cloud environment, the user is provided with the option of new registration and re-execution regarding the login operation. When the user registers or logs in, there is a lack of memory and monitoring related to the username and password. Here RBAC uses datacenter to authorize and exchange their works to maintain and monitor the access control activities properly (Mei-Yu et al., 2014). In the web service domain, whenever large information is floated, there will be a compromise in security. So, Rafae (2005) discusses both context and capability based control scheme where they have identified the lack of security aspects in web services. To make the trusted access control model, Rafae (2005) proposed an eXtensible Markup Language (XML) based role-based access control (X-RBAC) which integrated both security and context-based access control accessing through policy-based service framework. The work had included XML based secure service to improve security based on access control RBAC (Rafae et al. 2005).

Jacques takes the concept of RBAC workflow systems to propose a new RBAC model which provides permission service based on the workflow component. To provide authorization and authentication, RBAC model is in cooperated for controlling and systematic overriding constraint to perform control access (Jacques et al. 2003). Related to application-based services, Moon et al. discuss the application of health care where every person uses to get their status anywhere and anytime. Here to address the issue of improving quality and cost through security, the author proposes an RBAC based security model to protect privacy and improve security which includes all information related to health care (Moon et al. 2015).

Still, access control based web services use several other inter-disciplinary techniques to process the model. Chun et al., focus on the BPEL with the integration of control flow construction like sequence, branching, synchronization, etc. Here the author has taken the kernel framework based on Petri net structures to analyze the properties of garbage collection, deadlock and life lock, etc., during the interaction process. The proposed business process execution language model uses structural analysis based on the variety of question along with the answer (Chun et al., 2007).

Esmaeil and Mohammad (2009) discuss the work related to the solutions to be offered based on dependable web services and categorized into fault tolerance and design diversity. Here is proposed a new WS-BPEL architecture which depends on the design pattern of dependable web services (Esmaeil \& Mohammad, 2009). This work gives new dimensions to provide access control to other services rather than providing services to the real-time application. On the other side, the authors are focusing on dependable web services based on control access model. Mohammad \& Esmaeil (2012) discussed proposing a solution for dependable services based on the increase in user demand. WS-BPEL model is proposed which categorizes the solution into fault tolerance and design diversity model based on access control model. Here the author has extended his services by applying the WS-BPEL with Stochastic Reward Net (SRN) to measure the dependability (Mohammad \& Esmaeil, 2012).

Here Chun et al., give the new form of business process execution language framework integrating with business process modelling notation based on graph-based oriented language. While mapping these two models, the author considers the challenges based on scientific view and proposes a new model by mapping the business process execution language framework along with business process modelling notation based on flow-based programming using parallelism (Chun et al., 2006). As all the works discussed so far are taking the challenges of security based on Authentication, Authorization and Accounting (AAA) service as role-based, access control works are dealing with a large number of information exchange between several entities. Zhuo et al. (2012) discuss the problem of security lack while providing access control to several entities that include owner, employee and service provider based on several activities. Here, the existing RBAC models to a new RBAC model are 
formulated by taking two entity categories owner and employee and to ensure privacy, authentication and authorization. New RBAC model is implemented in a cloud environment by taking three entities such as owner, employee, and service provider. After applying the cloud framework, security is getting enhanced (Zhuo et al., 2012).

Only with the RBAC model can be integrated with the cloud environment, but other than RBAC there are other role-based models. So, Zhu et al, discuss various RBAC, CoRABC, dRBAC models where all these models are categorized under role-based access. The author has considered the challenge of security while performing role provisioning. Here CoRBAC model eliminates the unnecessary construction of secure flow and multi-cache setting and user experience and the process gets improved (Zhu et al., 2011). As cloud computing is providing and giving access to private services among the users, still there is a security challenge is getting prolonged. The author has refined several entities such as employee, owner and service provider based on the applications which use the RBAC model (Wei et al., 2012).

Shinji et al. elaborate on the BPEL process activities as a heterogeneous large-scale process (Shinji et al., 2012; Auxilia et al., 2016). Monitoring is conducted on the message and its activity based on cloud services. Monitoring of large-scale process in heterogeneous environment plays a curial role in terms of system management. Till now there is no proper monitoring of large-scale process in the heterogeneous process and this happens when there is no interoperability among multiple vendors while performing monitoring process functions. The resultant monitoring process of a heterogeneous business process by this common audit-data (Anwar \& Imran, 2015; Auxilia \& Raja, 2016) considers the ontology model and role provisioning for healthcare application through access control. In the analysis, they verify the vulnerabilities in multi-tenancy by accessing the control policies but do not consider the security level of the information exchanged between the persons employed by the RBAC framework. Still, the concept of security prevails in all the domains like network (Arul et al., 2018; Ramkumar et al., 2018), data mining (Kannan \& Raja, 2016) rather than web services because information of large quantity is in existence while sharing or monitoring the information.

Thus, this domain seeks a fruitful solution that solves the data integrity issue thereby preserving trust and authenticity. In this paper, a new framework for cloud access control for role assignment based on the user behaviour is proposed and it inferred that our model is tamper proof through various analyses.

\section{C-BAC ROLE PROVISIONING FRAMEWORK}

\section{Role Classification Based on Access Control}

Healthcare Application: Roles provisioning are deployed in a hospital application for several service organizational units such as pharmacy, physician, laboratory and shipping. Here the hospital process includes operations which are served as the laboratory services. It allows lab assistant to perform certain operations in the system to collect the different types of blood samples which are labelled in the collected blood. Then the doctor leaves the comment in the tested blood sample results, which is referred to as test referral operation. After the comment from the doctor, the blood test results are scheduled for shipping to the patient concerned from the lab employee, which is referred to as update record operation. Then simultaneously, pharmacy service gets activated which allows the physician to send the medical prescription of the particular patient to the hospital pharmacy which is referred to as send prescription operation. Finally, the prescribed medicines are sent to the patient which is scheduled by the pharmacy employee as a delivery operation. The role provisioning operation process for hospital application is represented in Figure 1. 


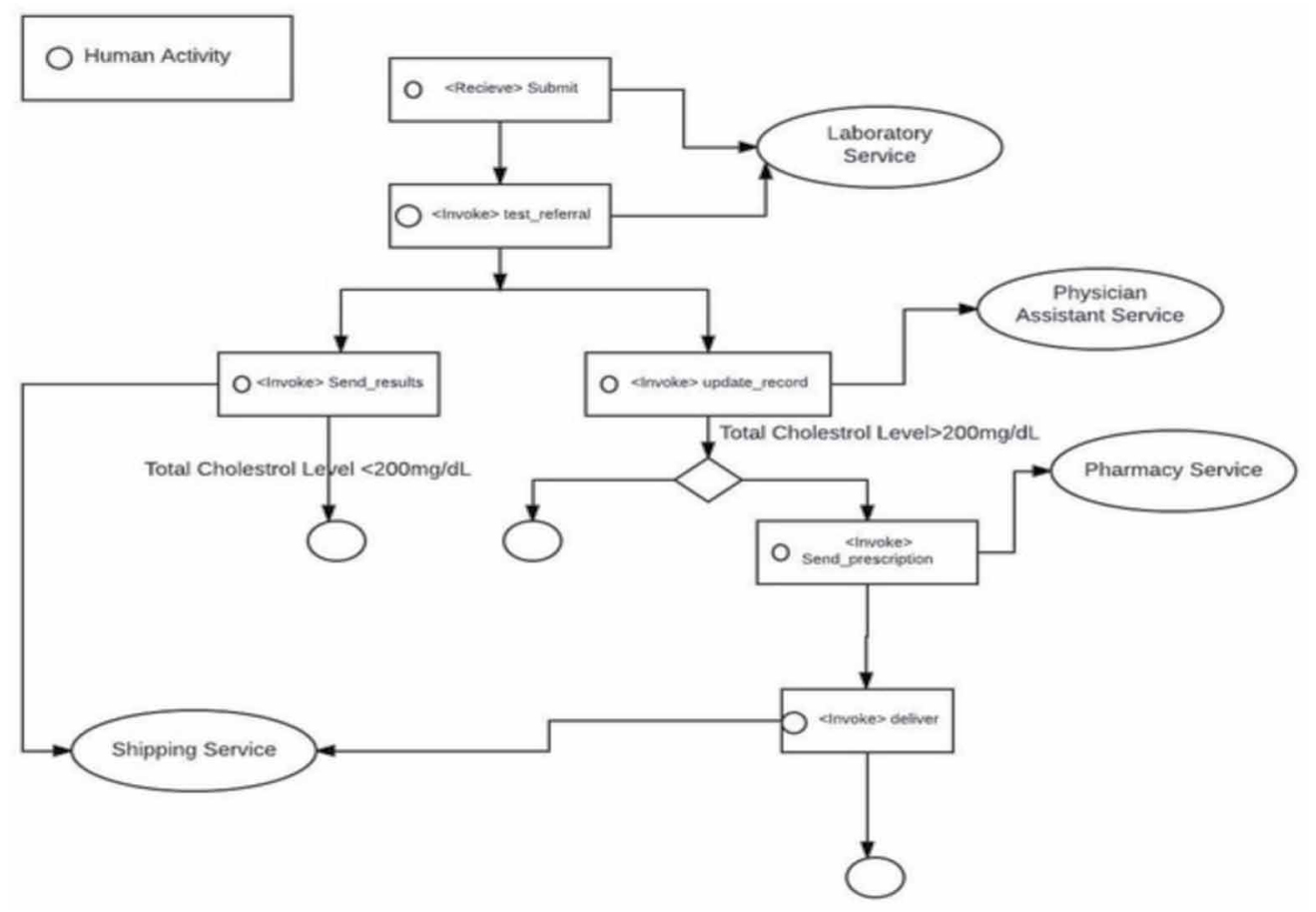

\section{ROLE ASSIGNMENT BASED ON ACCESS CONTROL METHODS}

To perform restriction for accessing the system to the authorized users, RBAC approach is used. Performing this kind of activity which is called permission activity helps in performing certain operations to be assigned to the particular persons who are assigned with certain roles. In recent trends, there are new approaches which include, Mandatory Based Access Control (MBAC) and Discretionary Based Access Control (DBAC). Compared to the other approaches in access control, these MBAC and DBAC will not assign permission to the persons directly to the role unless they are assigned with the role. These access control methods follow three rules to assign permission to the employee after assigning the role. First, execute the transaction or any other operations only if the person is assigned with the role which is identified as role assignment. Then the role person is assigned with that operation/subject. Based on the role assignment, persons are ensured to take that role operation which is given authorization and it is identified as role authorization. Finally, execute the transaction/operation which is performed by the authorized person and it is identified as transaction authorization. Both role assignment and role authorization ensure that the employee who has the authority will only execute the particular transactions.

RBAC is the superset of Lattice-Based Access Control (LBAC) with the concept of role hierarchy and its constraints.

The hospital organization has certain role hierarchies to which, specific roles are assigned and their transactions are authorized. Access methods based on role provisioning (Hassan et al., 2007) assign permission to the assigned role where the users are given that appropriate role as the administration which makes the user suitable for the system organization. Considering the hospital system management, patient records, prescriptions, images, results, etc., are identified as objects, whereas administrator, medical students, patient, nurse, physician etc., are identified as roles in the hospital. The roles assigned in this system are fixed and group-based which helps in reducing the 
complexity of the security-based operations. In the health care based system, user authorization is very important. Access methods based on role assignment provides a naming assignment, many to many relationships between several individuals' description and their rights. Based on the frequent function usage of the employee instead of on the ownership of data in the organization, access method provides control towards the user authorization and also to protect the resource which is used by the employee. As the roles assigned are not assigned with issuing permission, it also interrelated with other roles assigned to some other with their respective permission. The access method operations are extended to the role hierarchy as it represents the user responsibility and their specialized role. The role hierarchy is limited in assigning the redundant permission among the roles and also provides permission to make the work easier for the role of administrator. The role hierarchy of hospital diagnosis system is represented in Figure 2.

- User: A user is a person or an autonomous agent;

- Roles: A role is a kind of job which has several functions like a physician, shipping, etc. are cooperated into it and it is associated with various permission to make it the resources/data secure.

Permissions (objects and operations) -Permission includes objects, permission, etc., which gives authority and rights to access and perform the operation/activities especially to protect the resource and perform a certain task based on the system.

In the C-BAC process life cycle, users are enrolled for a particular role in certain applications as represented in Figure 3 and providing the services to a particular role as represented in Figure 4.

General Condition:

"R $\rightarrow$ Condn1, Condn2 ...CondnN", $\mathrm{n}>=1 "$

$\mathrm{R} \rightarrow$ Role.

Condn1, Condn2 ...CondnN $\rightarrow$ Attribute Conditions (based on Figure 1)

Figure 2. Role-based access control model

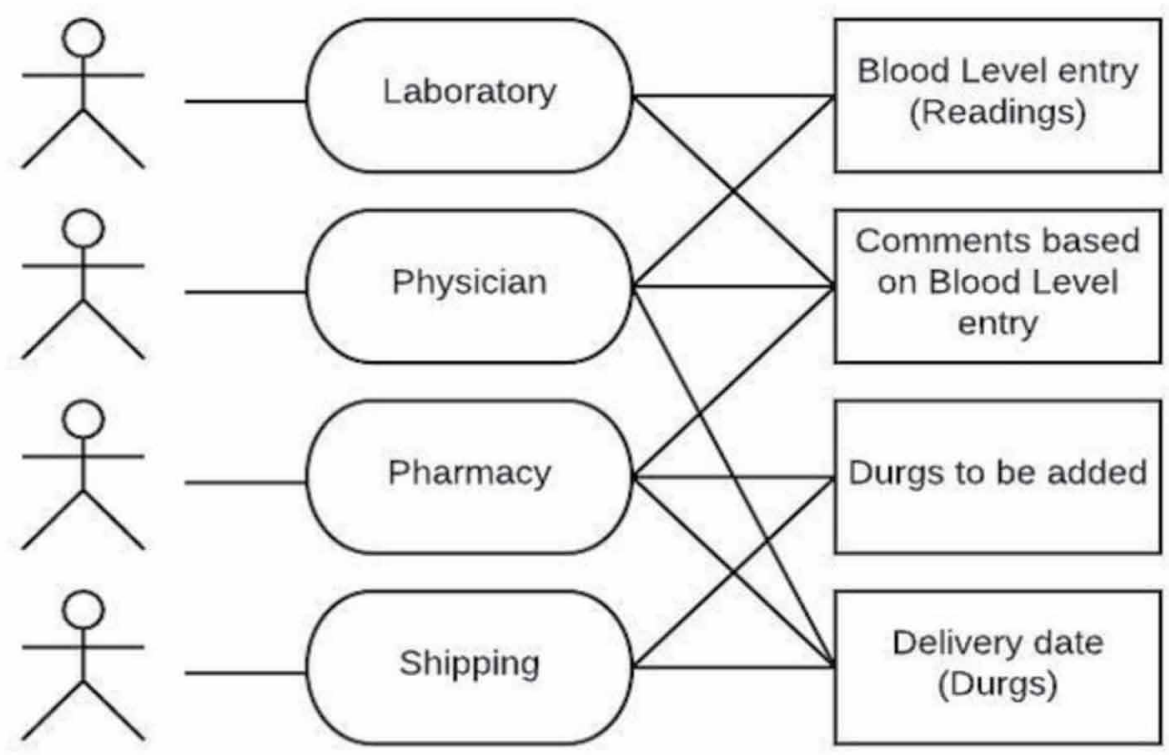




\section{Figure 3. User classification and enrolment process}

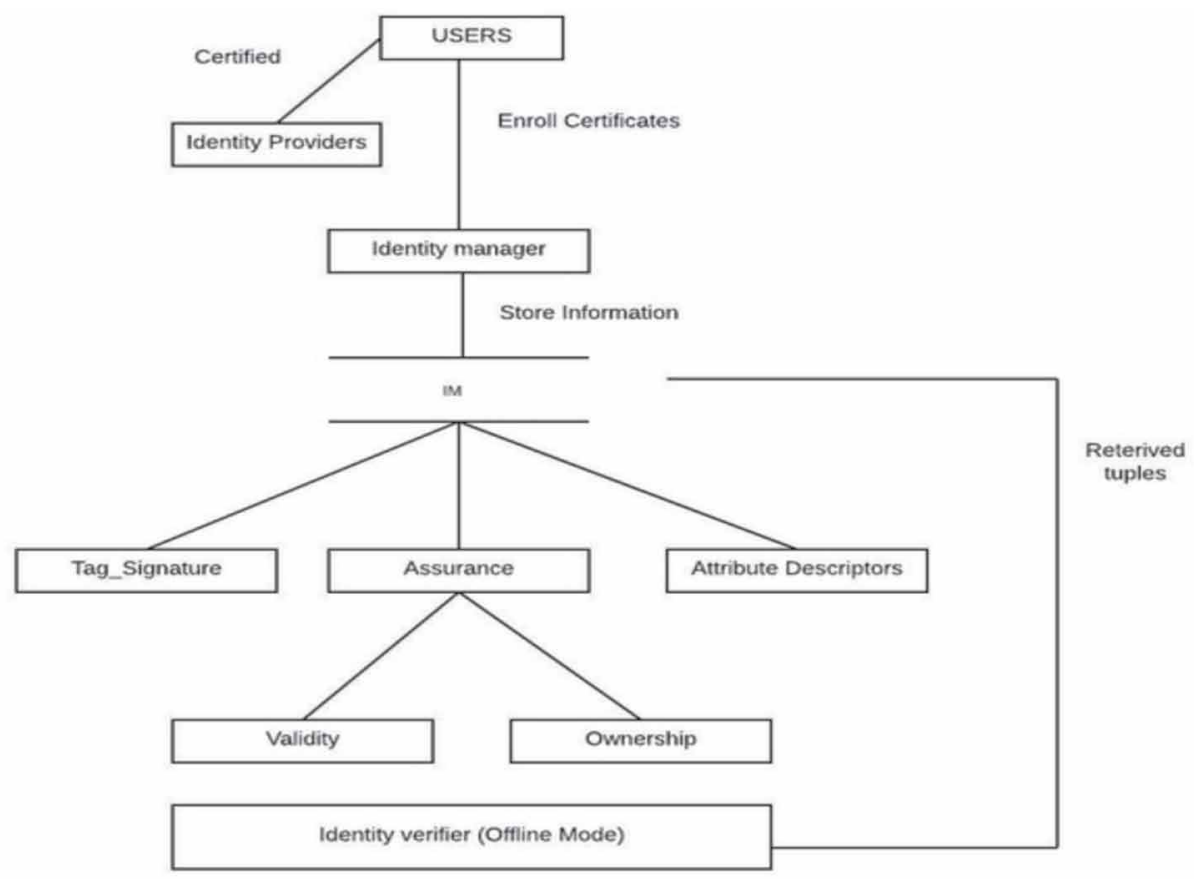

In the user classification and enrolment process, initially the users are classified and each user has to enrol the access control based on the C-BAC framework. First, the user has to be certified by the provider, who provides the control access. For enrolment of a certificate, IM (Identity Manager) has to store the information regarding the access control of a particular user, i.e., information tuples like Tag/Signature, identity attributes. Consider the example for healthcare which contains several attributes like birth-date, employmentID, employee security number etc., Assurance or authentication, whether the user is valid or invalid to these access policies. If valid then the user is allowed to access and control the policy based on authentication. The user enrolment process can be adapted to either online or offline (Ehsan \& Nikbakhsh, 2018).

In role provisioning process, client enforcement takes place. Initially, the user requests the enforcement point for a certain activity, by selecting the authorized role for the user/client. For the selected authorized role, there are certain policies ' $\mathrm{R}$ ' $\leftarrow$ Cond1, Cond2, .. Condn, which helps to compute the sets having conditions or no conditions attributes. For No Condition policy, AgZKPK protocol is used and for condition policy, OCBE protocol is used. For the policies selected, check whether the attributes satisfy to carry out the AgZKPK or OCBE protocol:

1. Aggregate Zero Knowledge Proof Knowledge Protocol (AgZKPK): In this protocol, aggregated zero knowledge is defined as an interactive method where one party has to prove mathematically when the statement is true without illuminating anything other than the reliability of the statement. Using the protocol discussed above, the restriction is performed on the non-trusted person who cannot learn anything about the protocol, cannot cheat the verifier process, verifier process cannot cheat the evidence and verifier cannot make the evidence to be showcased the third party. The above-said discussions are helpful in securing the process and the resources used; 
2. Oblivious Commitment Based Envelope Protocol (OCBE): The protocol helps in ensuring the receiver ' $R$ ' to decrypt the message sent by the sender ' $S$ ' only if the dedicated value satisfies the condition given by ' $S$ ' by means of the control policy. In this process, ' $S$ ' knows nothing about the dedicated value which is represented as $=, !=,>, \geq,<$ and $\leq$.

After verification, the user may or may not take the decision to assign the role or not. When the user is assigned with the role, role certificate is issued where the user is identified as an authorized one.

PEP is a logical entity which is placed on the server and enforces certain policies related to admission control, i.e., strict as to whom to enter and whom to exit. It also applies decision policy to response whenever the request from the user is assigned which helps in accessing the resource placed in the system.

PEP is an element which will manage based on the policy. In the policy, the user will try to access the file/resource in the computer which is placed as a network or server to manage based on the rules. PEP will take certain decision to describe the features to other users in the system. In certain case, PEP will take the decision whether to authorize the user or not into PDP based on the user attributes description. Only the applicable policy of the user concerned is stored in the system, which is then analyzed by PDP. Then PDP will take and return the decision which will help the PEP to know whether the user has the permission to authorize the access based on the resources. Finally, based on the above process the C-BAC framework is developed to make an effective and secure resource exchange based on the user and admin which is represented in Figure 5.

In providing security to the C-BAC framework, existing Advanced Encryption Standard (AES) algorithm is used, which does not provide much security and authentication. In addition to the above discussed in the proposed framework, Rivest, Shamir and Adleman (RSA) are used which is trusted as the security standard algorithm for securing the resource in recent days (Anwar \& Imran, 2015) which will perform encryption and decryption. RSA algorithm follows public key cryptography which

Figure 4. Role provisioning process

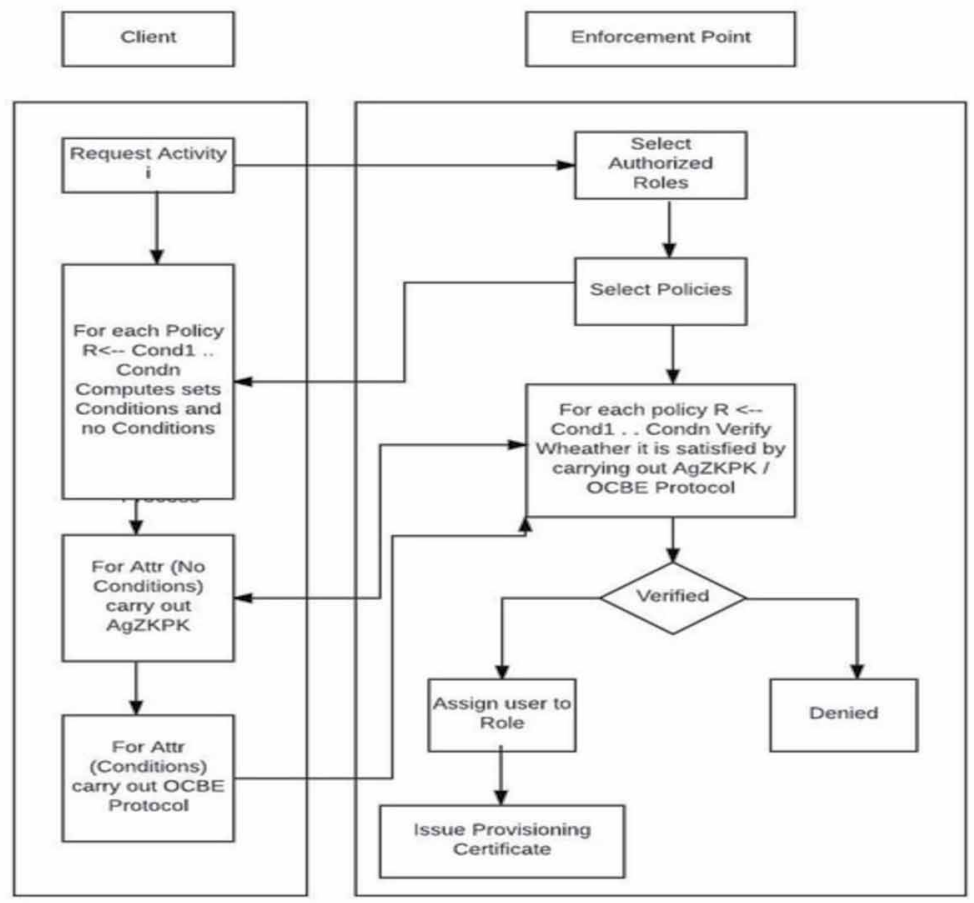


Figure 5. C-BAC framework

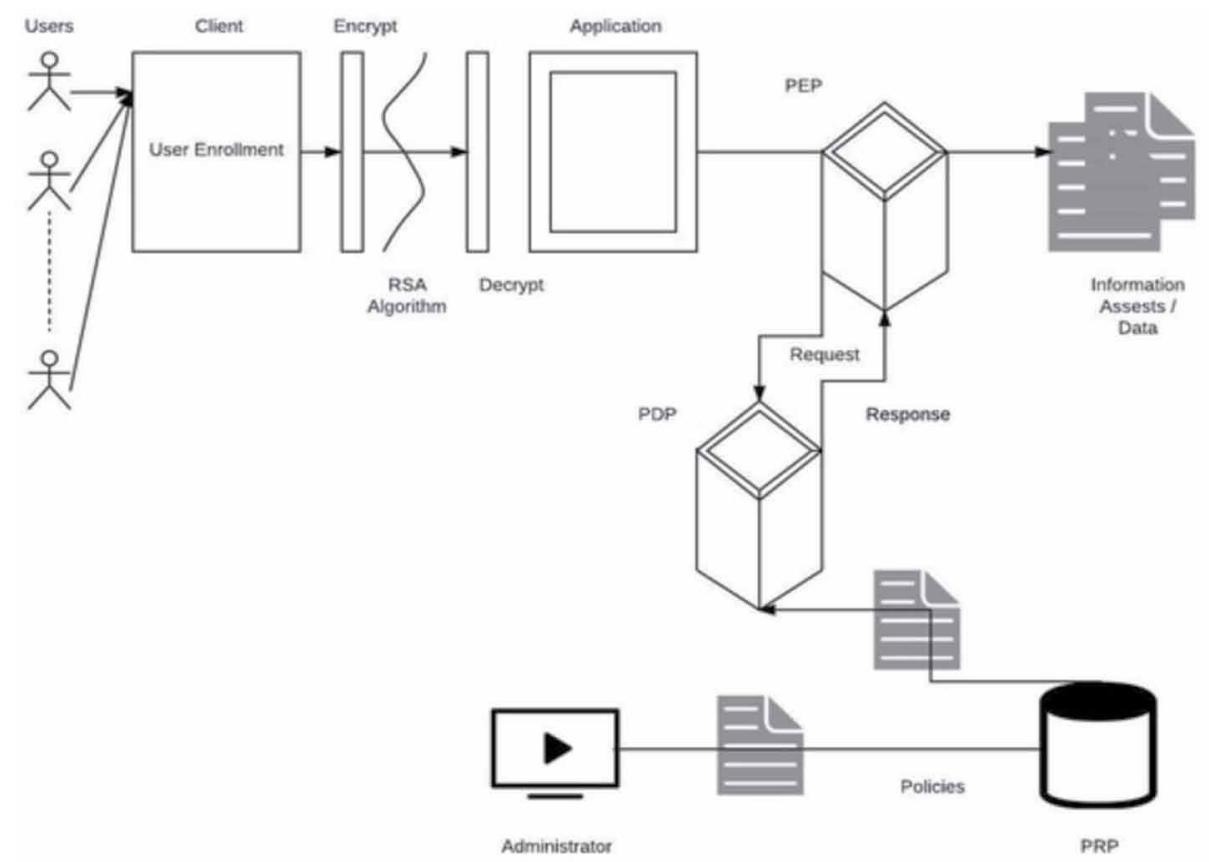

is widely used in the electronic-based application. It will secure the resource with a sufficient number of keys where RAS uses several varieties of bits to make a secure resource by using key generation.

Initially in RSA algorithm, select randomly a, b, c where a is a prime number which should be less than the product of $b$ and $c$. Using the random selection, Compute, $d=\left(b^{*} c\right)-a$; $e=(a * d)+c ; f=(a 2 * d)+b$. Using the random selection and computation of $\mathrm{d}$, e, and $\mathrm{f}$. Calculate public key, i.e., $n=\left[\left(e^{*} f\right)-a\right] / d$. Using the Public Key $\{e, n\}$, Encryption process takes place and after generating the public key $\{e, n\}$, using plain text ' $\mathrm{M}$ ', Calculate encrypted text ' $P$ ' $=M^{*} e(\bmod n)$ to get the Encrypted text. To generate Private Key, set the condition as $h \operatorname{Pr} i * r(\bmod n) \equiv 1$ and $0 \leq r \leq n$, where $h \operatorname{Pr} i=$ private key. To calculate $k=r * t$, where private key $\langle k, n\rangle$. The Decryption Process helps to get back the original message, compute $O=P^{*} k(\bmod n)$ to Decrypt the text.

\section{Bilinear Mapping}

Let B1 and B2 be two groups and the additional group represented as BT. Where, $\left|B_{1}\right|=\left|B_{2}\right|=\left|B_{T}\right|$ :

$$
B_{e}=B_{1} x B_{2} \rightarrow B_{T}
$$

1. Bilinear: $u \in B_{1}, v \in B_{2}$ and $a, b \in Z, e\left(u^{a}, v^{b}\right)=e(u, v)^{a b}$; 
2. Non-degenerate: $e\left(B_{1}, B_{2}\right) \neq 1$.

\section{Modified RSA}

In the modified RSA, Input messages $M=\left\{m_{1}, m_{2}, m_{3}, \ldots m_{t}\right\}$ and equivalent signatures $S=\left\{s_{1}, s, s_{3}, \ldots s_{t}\right\}$ are considered and each signature using bilinear mapping is given by:

$$
\left(s_{l, t}\right)=\prod_{i=1}^{t} S_{i}(\bmod n)
$$

After computing the signature (2), all the input messages are mapped with a hash function given by:

$$
\left(s_{l, t}\right)^{e}=\prod_{i=1}^{t} h(M)(\bmod n)
$$

After signature (3), verification for all the messages with the batch order is given by:

$$
\left(\prod_{i=1}^{t} S_{i}\right)^{e} \equiv \prod_{i=1}^{t} h(M)(\bmod n)
$$

In the case of modified RSA algorithm procedure, Message $M=\left\{m_{1}, m_{2}, m_{3}, \ldots m_{t}\right\}$ and $\theta$ are considered and to determine the output as $O=\left\{o_{1}, o_{2}, o_{3}, \ldots o_{t}\right\}$ where:

$$
\left(\prod_{i=1}^{t} S_{i}\right)^{e}=\prod_{i=1}^{t} H(M)(\bmod n)
$$

Compute Output 'O' using (5) as:

$$
o_{t}=\left(\prod_{i=1}^{t-1} S_{i}\right)^{-1} * X(\bmod n)
$$

Then the message digest (MD) from this integer is extracted and they are independently computed based on MD of the signed information. Only if both messages digest of both sender and receiver messages are identical, then the signature is validated.

\section{IMPLEMENTATION AND PERFORMANCE ANALYSIS}

\section{Developing C-BAC Framework Based on Workflow}

Workflow is a "series of distinct programming steps or phases. Each step is modelled in the workflow as an Activity" represented in Figure 6. For the implement of C-BAC framework in the platform of workflow, the .NET framework is used which will perform a certain activity by writing text into the 


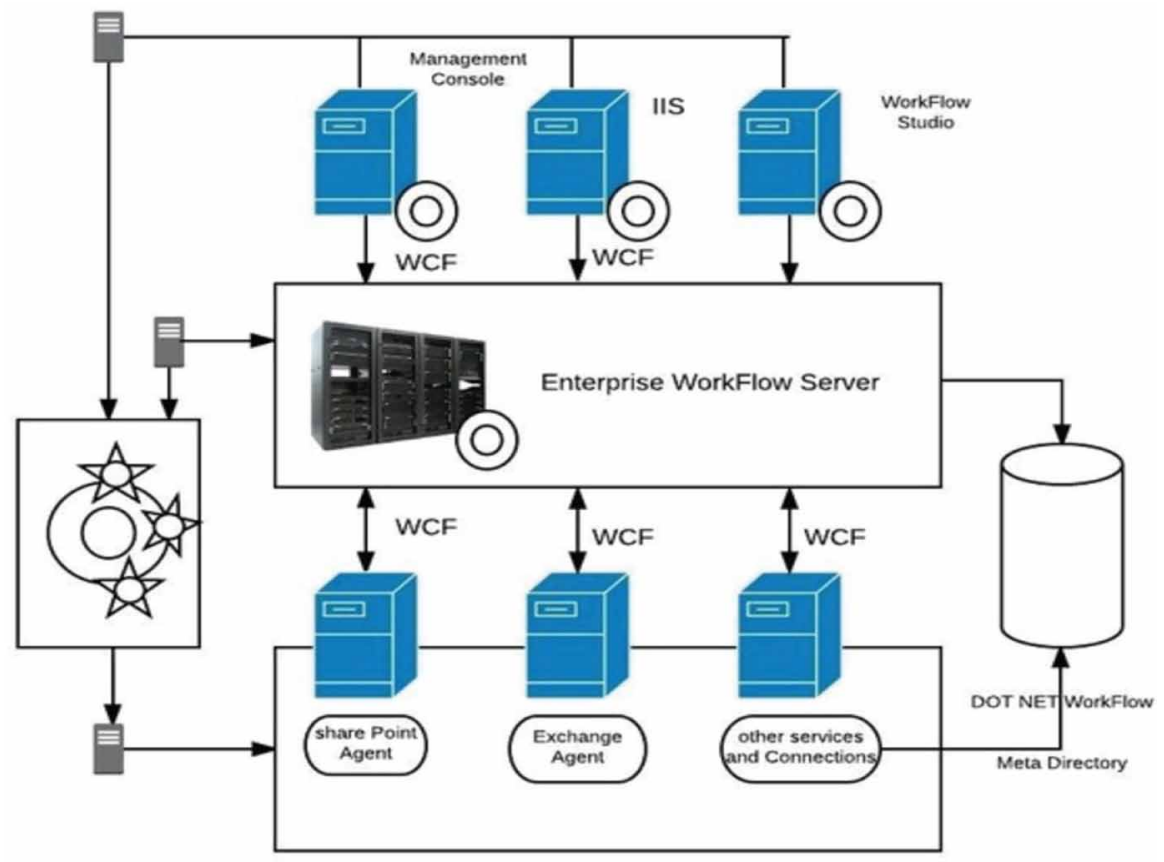

console. Certain activity can be customized by adding certain functionality. The activities performed based on the system can be visualized in the workflow framework by using the workflow designer surface.

The workflow process is categorized based on multi-tier which includes a smart client application, web site, workflow service and data layer. Initially, the applicant has to submit the application by means of client application through window forms using workflow service. Then screening the applicant takes place using workflow service by calling in terms of education service. Unless the screening gets passed, the applicant will receive an email along with the link of the web site followed by screening. Then the review results are called by the workflow service to check whether he is passed or not. Finally, the applicant will be again notified through email to call him for the client application.

The developed workflow for hospital medical diagnosis is represented in Figure 7.

The created workflow is assembled using dot Net programming either using C\# or VB.Net. In the workflow framework, workflow designer is used to representing the assembled activities by dragging from the toolbox. Otherwise, when the workflow process is assembled by means of a code, then it has the collection of parent activity in terms of sequence or flow chart. This parent activity gets executed and run by using the workflow framework. The workflow originally comes from the root activity by the host application and it uses certain out-of-box activities including:

1. Workflow process uses Do While statement to process the activity;

2. Workflow uses flow decision representation;

3. Information like activities is sent by using WCF;

4. Basic activities and tack to be executed using write line;

5. While performing custom activities, users will create a CLR object which is derived from the class system and also provides a function declaration and allows the developer to define the behaviour of activity which is to be executed. The custom activities will have certain benefits based on the designer which is associated to enhance the visual experience of the visual studio. 


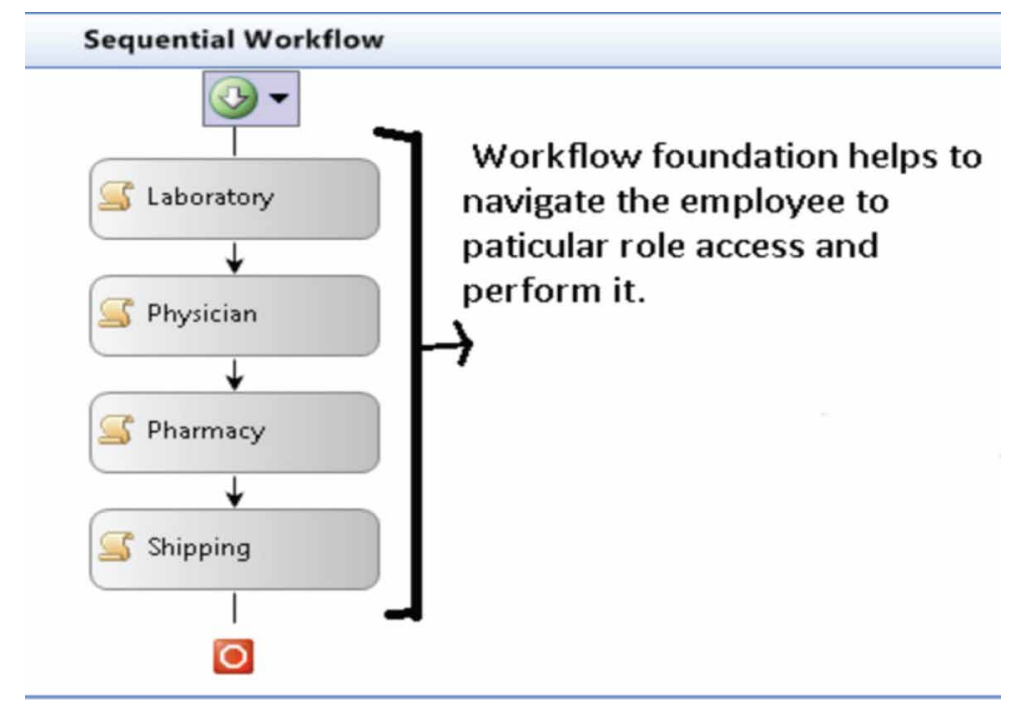

\section{ANALYSIS OF THE DEPLOYMENT OF SECURITY ALGORITHMS IN C-BAC FRAMEWORK}

In this implementation, role provisioning integrated with Workflow Process is further integrated with the C-BAC model. Existing BPEL is used and it is more expensive than Workflow. Workflow Foundation is not expensive and well suited to .NET Framework. Then MYSQL is used as the database to store identity attributes and information of an employee.

The analysis is done based on the security level for several security algorithms in the C-BAC framework. As compared to the existing Access Control with the multi-tendency framework (Anwar \& Imran, 2015) with AES and RSA. C-BAC framework with RSA provides better security based on the number of employees with the data handled by a particular employee as shown in Figure 8.

In Table 1, the cost with respect to the number of resources, i.e., data utilized for the services is calculated. Based on the analyses, we have determined that the proposed C-BAC system performs better with less cost than the existing algorithms such as E-RBAC and RBAC with multi-tendency as same as in Table 1, Figure 9 determines the execution time based on the number of resources utilized related to the cost. Based on the analyses, the proposed C-BAC takes less execution time compared with the existing algorithms are inferred.

Slack value is nothing but the delay in the task which is performed with respect to the number of resources getting utilized and is illustrated in Figure 10. Based on the analysis, the proposed C-BAC gets lesser slack value compared with the existing ones are determined.

The Performance overhead keeping a smaller portion for performance overhead until the number of requests reaches up to 50. The performance overhead gives the most portion of the role request overhead. Figure 11 shows the calculation of total network traffic overhead as the number of role requests increases from 10 to 50 .

\section{CONCLUSION}

In the proposed C-BAC, several conditions are applied based on identity attributes to assign a role to an employee by using several protocols and to protect the privacy information of the employee. The security strengths of the existing access control framework and the proposed framework C-BAC are 
Figure 8. Role enrolment performance graph

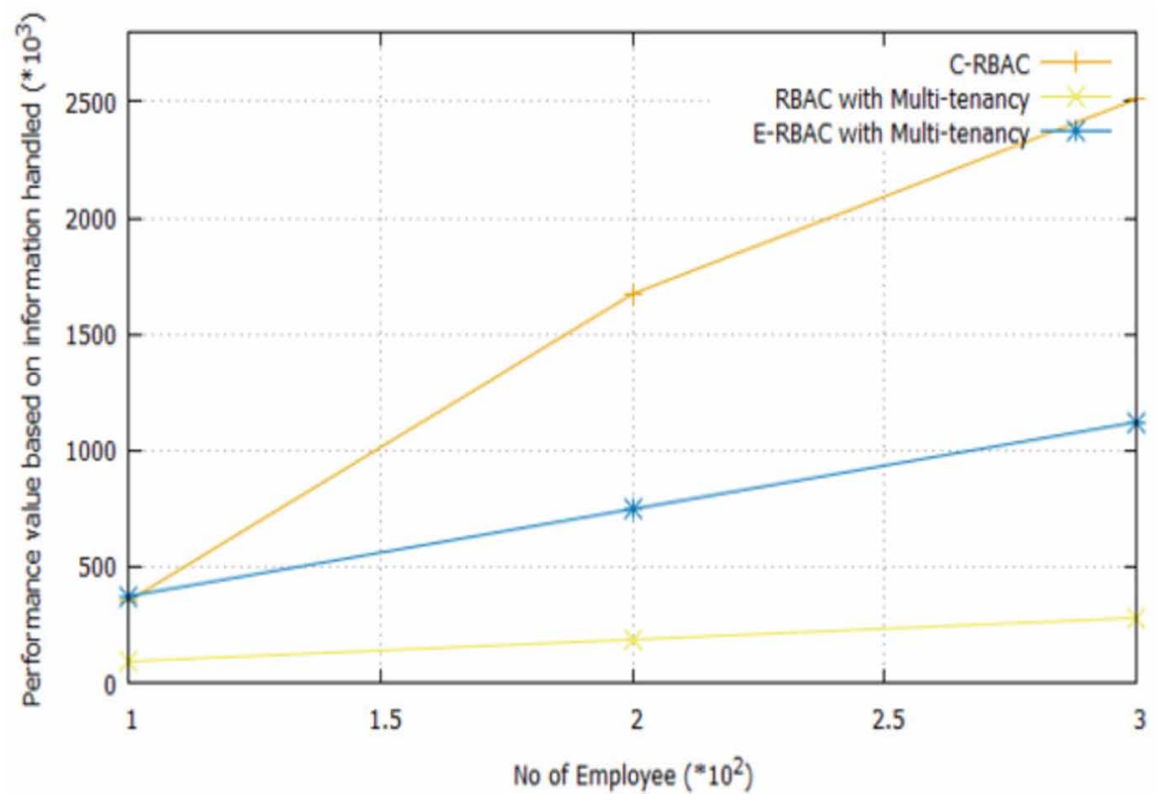

Table 1. Cost vs. Number of resources

\begin{tabular}{|c|c|c|c|}
\hline \multirow[b]{2}{*}{ No of Resource } & \multicolumn{3}{|c|}{ Cost $*\left(10^{2}\right)$} \\
\hline & C-RBAC & $\begin{array}{c}\text { E-RBAC With Multi- } \\
\text { Tenancy }\end{array}$ & RBAC With Multi-Tenancy \\
\hline 10 & 0.238 & 0.245 & 0.252 \\
\hline 20 & 0.270 & 0.300 & 0.315 \\
\hline 30 & 0.340 & 0.355 & 0.420 \\
\hline 40 & 0.350 & 0.415 & 0.425 \\
\hline 50 & 0.420 & 0.455 & 0.465 \\
\hline
\end{tabular}

measured and compared based on several parameters such as information handled, cost, execution time and slack deadline. In this analysis, the slack deadline in the cloud which represents how much the task (or) activity is getting delayed, i.e., time window and as compared to existing systems such as, E-RBAC and RBAC with multi-tendency with deadline above 500, the proposed system gets the least delay of below 100 in deadline. With respect to security based on a large number of data handled by the particular employee, proposed C-BAC framework provides $80 \%$ increase in providing better security than the existing systems with $40 \%$. In future, improved security by applying policies to the web service by developing a new framework for several other applications can be applied. 
International Journal of System Dynamics Applications

Volume 9 • Issue 1 • January-March 2020

Figure 9. Number of resources vs. Execution time

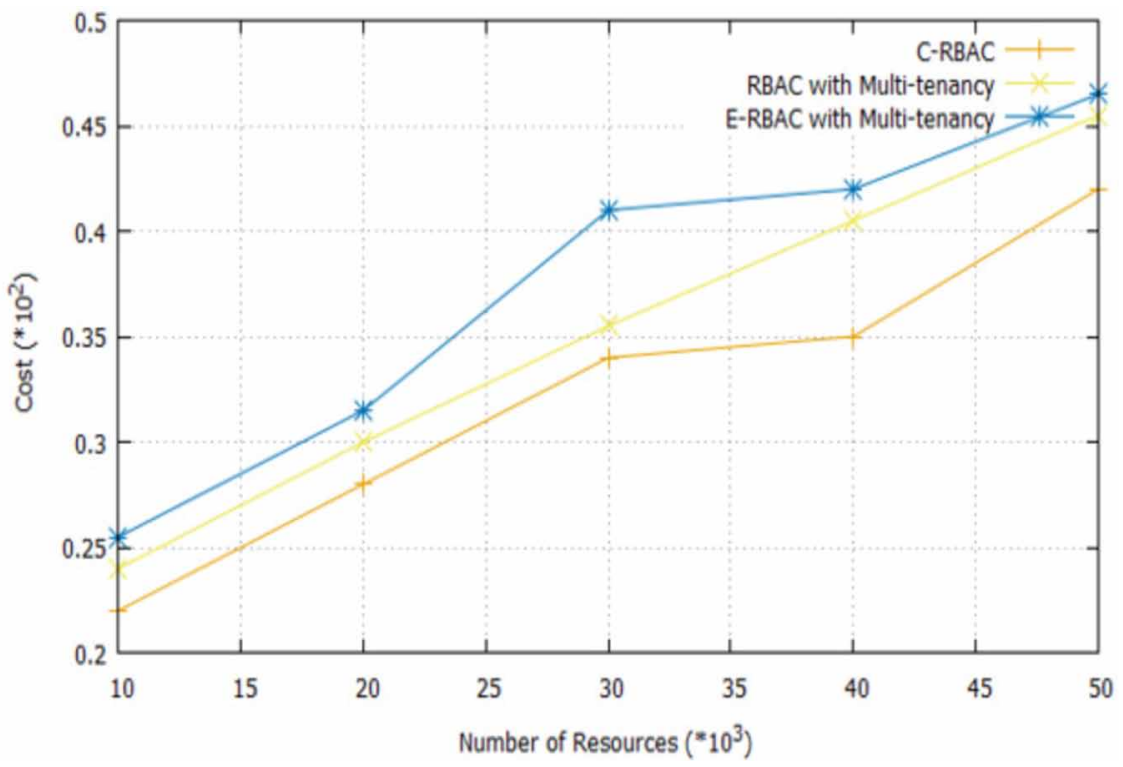

Figure 10. Number of resources vs. Slack deadline

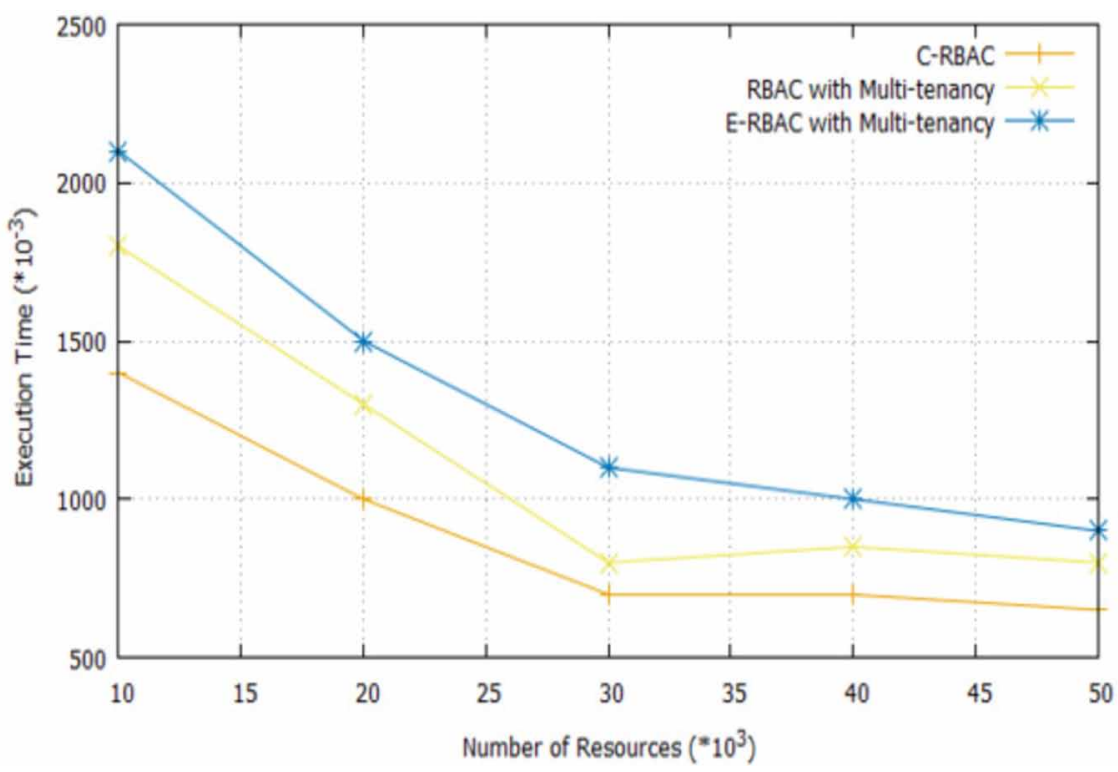


Figure 11. Network traffic based on performance overhead

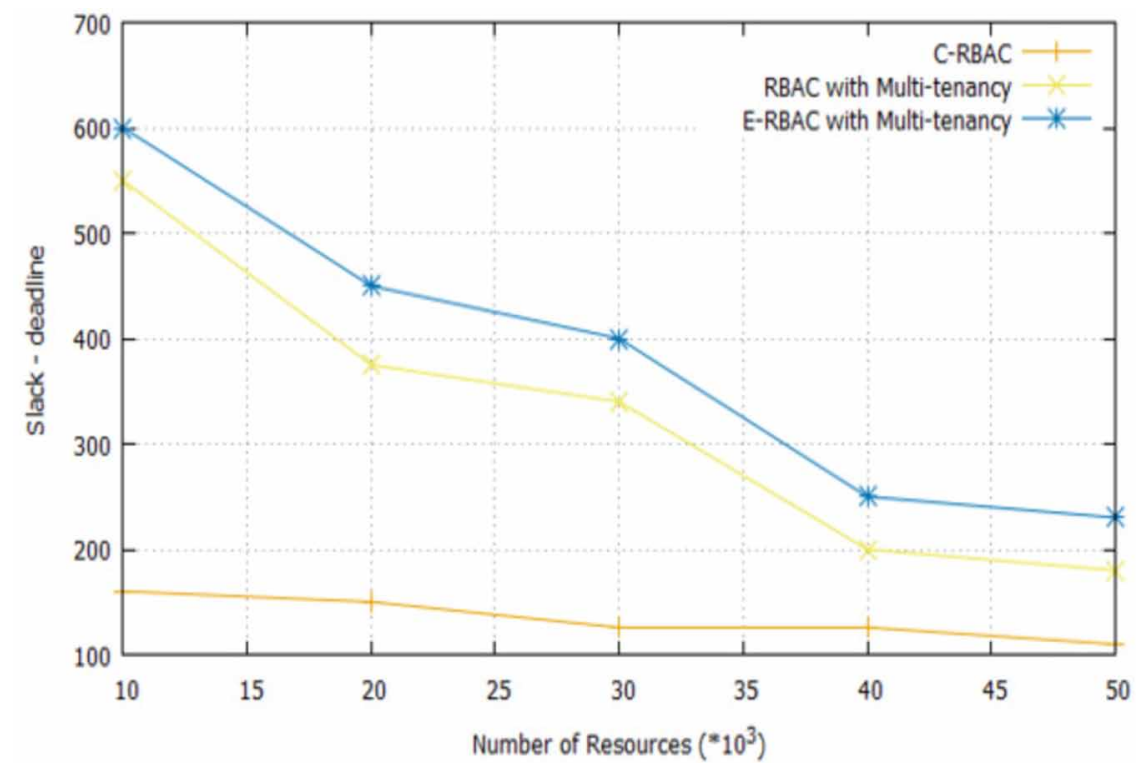




\section{REFERENCES}

Anwar, M., \& Imran, A. (2015). Access Control for Multi-Tenancy in Cloud-Based Health Information Systems. In Proceedings of the 2nd IEEE International Conference on Cyber Security and Cloud Computing. IEEE. doi:10.1109/CSCloud.2015.95

Arul, R., Raja, G., Bashir, A. K., Chaudry, J., \& Ali, A. (2018). A Console GRID Leveraged Authentication and Key Agreement Mechanism for LTE/SAE. IEEE Transactions on Industrial Informatics, 14(6), 2677-2689. doi:10.1109/TII.2018.2817028

Auxilia, M., \& Raja, K. (2016). Migrating Bank Data to Cloud: Threats and Mitigation. International Journal of Applied Engineering Research, 9(24), 28879-28902.

Auxilia, M., \& Raja, K. (2016). Ontology Centric Access Control Mechanism for Enabling Data Protection in Cloud. Indian Journal of Science and Technology, 9(23), 1-7. doi:10.17485/ijst/2016/v9i23/95148

Brian, J. G. (2018). Application of system engineering to project management: How to view their relationship. International Journal of System Dynamics Applications, 7(4), 22.

Chandrashekar, J., Gangadharan, G. R., \& Rajkumar, B. (2017). Computational Intelligence Based QoS-Aware Web Service Composition: A Systematic Literature Review. IEEE Transactions on Services Computing, 10(3), 475-492. doi:10.1109/TSC.2015.2473840

Chun, O., Eric, V., Wil, M. P., Vander, A., Stephan, B., Marlon, D., \& Ter, H. et al. (2007). Formal semantics and analysis of control flow in WS-BPEL, Science of Computing Programming. Elsevier Publications, 67(23), 162-198.

Chun, O., Marlon, D., Arthur, H. M., Ter, H., Wil, M. P., \& Van, D. A. (2006). From BPMN Process Models to BPEL Web Services, In Proceedings of IEEE International Conference on Web Services (ICWS'06). IEEE. doi:10.1109/ICWS.2006.67

Ehsan, G. G., \& Nikbakhsh, J. (2018). A system dynamics model for studying the policies of improvement of chicken industry supply chain. International Journal of System Dynamics Applications, 7(4), 18.

Esmaeil, N., \& Mohammad, A. A. (2009). A design pattern for dependable web services using design diversity techniques and WS-BPEL. In Proceedings of International Conference on Innovations in Information Technology (IIT). Academic Press. doi:10.1109/IIT.2009.5413635

Federica, P., Rodolfo, F., Yuqing, S., \& Elisa, B. (2008). Authorization and User Failure Resiliency for WSBPEL Business Processes. In Proceedings of the International Conference on Service-Oriented Computing, ICSOC (pp. 116-131). Academic Press.

Feldiansyah, B. N. (2018). Public policymaking framework based on system dynamics and big data. International Journal of System Dynamics Applications, 7(4), 16.

Hai-bo, S., \& Fan, H. (2006). An Attribute-Based Access Control Model for Web Services. In Proceedings of Seventh International Conference on Parallel and Distributed Computing, Applications and Technologies PDCAT '06. Academic Press. doi:10.1109/PDCAT.2006.28

Hassan, T., Morteza, A., \& Rasool, J. (2007). Trust-Based User-Role Assignment in Role-Based Access Control. In Proceedings of IEEE/ACS International Conference on Computer Systems and Applications, AICCSA 2007. IEEE. doi:10.1109/AICCSA.2007.370725

Hooman, A., \& Seyed, B., E. (2019). Modelling and Investigating the Economy and Production Structure of Iran Public Theater: A System Dynamics Approach. International Journal of System Dynamics Applications, 18(1), 19.

Ilia, P., \& Rizos, S. (2016). Cost-Efficient CPU Provisioning for Scientific Workflows on Clouds. In Proceedings of the International Conference on Grid Economics and Business Models. Springer.

Jacques, W., Paulo, B., \& Akhil, K. (2003). W-RBAC - A Workflow Security Model Incorporating Controlled Overriding of Constraints. International Journal of Cooperative Information Systems, 24(4), 455-485. 
Jiangtao, L., \& Ninghui, L. (2006). A Construction for General and Efficient Oblivious Commitment Based Envelope Protocols. In Proceedings of International Conference on Information and Communications Security (pp. 122-138). Academic Press.

Jonathan, C., \& Dusit, N. (2017). Joint Optimization of Resource Provisioning in Cloud Computing. IEEE Transactions on Services Computing, 10(3), 396-409. doi:10.1109/TSC.2015.2476812

Kalliopi, K., Nick, B., \& Guido, G. (2016). A policy-based B2C e-Contract management workflow methodology using semantic web agents. Artificial Intelligence and Law, 24(2), 93-131. doi:10.1007/s10506-016-9177-2

Kannan, K., \& Raja, K. (2016). Decision Making Process For B2C Model Using Behavior Analysis With Big Data Technologies, Indian Journal of Science and Technology. The Indian Society of Education and Environment, 9(24), 1-6.

Lazaros, M., \& Ahmad, T. A. (2017). New Discrete Time 2D Chaotic Maps. International Journal of System Dynamics Applications, 6(1), 28.

Mei-Yu, W., Shih-Fang, C., \& Yi, J. W. (2014). A Single Sign-On Mechanism with RBAC in Cloud Environment Multimedia and Ubiquitous Engineering. In Multimedia and Ubiquitous Engineering (pp. 323-328). Springer.

Mohammad, A. A., \& Esmaeil, N. (2012). A dependable web service architecture based on design diversity techniques and WS-BPEL. Iranian Journal of Electrical and Computer Engineering, 11(1), 1-10.

Moon, S. S., Heung, S. J., Yong, W. J., Bum, J. L., \& Seon-Phil, J. (2015). Constructing RBAC Based Security Model in u-Healthcare Service Platform. Scientific World Journal. doi:10.1155/2015/937914

Rafae, B., Elisa, B., \& Arif, G. (2005). A Trust-Based Context-Aware Access Control Model for Web-Services. Distributed and Parallel Databases, 18(1), 83-105. doi:10.1007/s10619-005-1075-7

Ramkumar, J., Gunasekaran, R., Ali, K. B., Chauhdary, S. H., Ali, H., \& Mohammed, A. A. (2018). Interference Mitigation Based on Radio Aware Channel Assignment for Wireless Mesh Networks, Springer -. Wireless Personal Communications, 101(3), 1539-1557. doi:10.1007/s11277-018-5776-4

Santosh, K. M., (2018). An Efficient Feed Foreword Network Model with Sine Cosine Algorithm for Breast Cancer Classification. International Journal of System Dynamics Applications, 7(2), 14.

Shinji, K., Yoshihiro, K., \& Yohsuke, I. (2012). Theoretical Considerations for maintaining the performance of composite web services. IEICE Transactions on Information and Systems, 65(11), 2634-2650.

Uwe, B., \& Tony, G. (2008). Software Technologies for Embedded and Ubiquitous Systems. In Proceedings of 6th IFIP WG 10.2 International Workshop, SEUS. Academic Press.

Ward, J., Alan, D., \& Adam, B. (2015). Workflow Partitioning and Deployment on the Cloud Using Orchestra. In Proceedings of the IEEE/ACM 7th International Conference on Utility and Cloud Computing (UCC). doi:10.1109/UCC.2014.34

Wei, L., Haishan, W., Xunyi, R., \& Sheng, L. (2012). A Refined RBAC Model for Cloud Computing, In

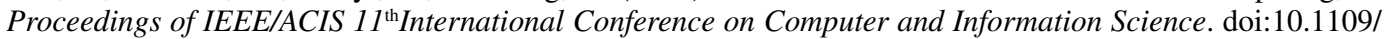
ICIS.2012.13

Yiqun, Z., Jianhua, L., \& Quanhai, Z. (2008). General attribute based RBAC model for web services. Wuhan University Journal of Natural Sciences. Springer., 13(1), 81-86. doi:10.1007/s11859-008-0116-2

Zhu, T., Liu, W., \& Song, J. (2011). An Efficient Role Based Access Control System for Cloud Computing, In Proceedings of IEEE $11^{\text {th }}$ International Conference on Computer and Information Technology. IEEE.

Zhuo, T., Juan, W., Ahmed, S., Kenli, L., \& Ruixuan, L. (2012). A new RBAC based access control model for cloud computing. In Proceedings of the 7th international conference on Advances in Grid and Pervasive Computing, GPC'12 (pp. 279-288). Academic Press. 
M. Auxilia is a Research Scholar in Sathyabama Institute of Science and Technology, Chennai. Her area of research is Cloud Computing. She is currently working as Associate Professor and Head in the Department of Information Technology at Christ College of Engineering and Technology, Puducherry, India since 2010. She had been conferred B.E Degree in the Department of Computer Science and Engineering in the year 2002 by Periyar University. She had been conferred M.E degree in the Department of Computer Science and Engineering by Anna University in the year 2008. She has got Diploma in J2EE from SISI. She has thirteen years of teaching experience in various reputed institutions. Her publications include 8 International Journals, 8 International Conferences, and 3 National Conferences, and 8 books for various subjects. She is an active and life member of Indian Society for Technical Education, and student member of Institute of Electrical and Electronics Engineers. Her area of interests includes Cloud Computing, Web Technology, Internet Programming, Computer Organization and Architecture, Web Services, SOA, and Data Structures.

K. Raja is working as a Principal and Professor at Dhaansih Ahmed College of Engineering, Chennai, India, since Sep 2016. He has completed his B. Sc (Mathematics) from Madras University, 1989, B.E (Computer Science and Engineering), M. E (Computer Science and Engineering) from Madras University, 2001, and Ph. D. degree in Knowledge-based systems from Sathyabama University, India, 2006. He is a life member of various professional bodies like Institution of Engineers India, Computer Society of India, and Indian Society for Technical Education, International Association of Computer Science and Information Technology, etc. He has more than two decades of teaching experience. He has published research papers in peer-reviewed, reputed 50 International Journals, 8 National Journals, 54 International (Singapore, Malaysia, Sri Lanka, Doha Qatar, and India), and National conferences. He has written 5 books in various fields. Under his Supervision of Research, 5 Research Scholars are Awarded the Ph.D. Degree.

K. Kannan is currently a Professor in the Department of Computer Science Engineering, AdhiSankara College of Engineering and Technology, Gudur, Nellore, Andra Pradesh State, India, since August 2019. He has completed his B. Sc., (CS) from Bharathiar University, 1991, M. Sc., (CS) from Bharathidasan University, 1994, M. E., (CSE) from Anna University, Chennai, India, 2008. He has conferred the Ph. D. Degree in Knowledge-Based Decision Support Systems, from Sathyabama University, Chennai, India. He is a Professional Member of ACM - Association for Computing Machinery and Life Member in ISTE - Indian Society for Technical Education. He has earned 20 Years of Academic Experience from various reputed educational institutions. He has published research papers in peer-reviewed, reputed 15 International / National Journals, and presented the papers in 25 International (Singapore, Doha Qatar, and India), and National Conferences. His areas of interest are Knowledge and Data Engineering, Decision Support Systems, Service Oriented Architecture, Web Technology Data Structures, and Data Warehousing. 